\title{
New and noteworthy lichenicolous and bryophylous fungi from the Ukrainian Carpathians
}

\author{
Valeriy V. Darmostuk ${ }^{1}$, Alexander Ye. Khodosovtsev ${ }^{1}$, \\ Jan Vondrák ${ }^{2,3}$, Olha Ye. Sira ${ }^{4}$ \\ ${ }^{1}$ Kherson State University, 27 Universytetska Str., 73000 Kherson, Ukraine. \\ E-mail: valeriidarmostuk@gmail.com \\ ${ }^{2}$ Institute of Botany of the Czech Academy of Sciences, Zámek 1, 25243 Průhonice, Czech Republic. \\ ${ }^{3}$ Department of Botany, Faculty of Science, University of South Bohemia, Branišovská 1760, \\ CZ-370 05 České Budějovice, Czech Republic. \\ ${ }^{4}$ V.N. Karazin Kharkiv National University, Svobody Sq. 4, 61022 Kharkiv, Ukraine.
}

\begin{abstract}
Nine species, Acremonium rhabdosporum, Arthonia digitatae, Bryocentria metzgeriae, Diplolaeviopsis cf. symmictae, Skyttea gregaria, Rhymbocarpus pubescens, Stromatopogon cladoniae, Tremella cetrariicola and Xenonectriella subimperspicua, are newly reported for Ukraine. Sphinctrina anglica is recollected for the first time since 1955. Parmelia saxatilis and Parmelina pastillifera are new host species for X. subimperspicua.
\end{abstract}

Keywords: Carpathian Biosphere Reserve, Gorgany Nature Reserve, Diplolaeviopsis, Stromatopogon, Xenonectriella

\section{INTRODUCTION}

The first mention of lichenicolous fungi in the Ukrainian Carpathians was published by Czech lichenologists in the first half of the last century (e.g. Servit \& Nádvorník, 1932; Servit, 1936). Further research was related to the project "Lobarion lichens as indicators of primeval forests in Carpathians (Ukraine)" supported by the Darwin Initiative in 1997. It was held in the Zakarpattia region and, as a result, supplemented data about lichens and lichenicolous fungi (Coppins et al., 1998). Records of lichens as well as lichenicolous species in the East Carpathians were summarized in the checklists by Kondratyuk et al. (2003). Furthermore, data about lichenicolous fungi in Ukraine and also in the Ukrainian Carpathians were included in the checklist by Darmostuk \& Khodosovtsev (2017). Further data are published by Darmostuk (2018), Malíček et al. (2018) and Nyporko et al. (2018).

During the two Ukrainian-Czech expeditions in May and August 2019, a few new and noteworthy lichenicolous fungi as well one bryophilous fungus were collected. The aim of this paper is to contribute to the new records of lichenicolous and bryophilous fungi in the Ukrainian Carpathians from Ivano-Frankivsk and Zakarpattia regions.

\section{MATERIAL AND METHODS}

The specimens were examined by standard microscope techniques using microscopes Optica-1 and MICROMED-2. Microscopical examination was done in water, $10 \% \mathrm{KOH}(\mathrm{K})$, Lugol's iodine solution, directly (I) or after pretreatment with $\mathrm{KOH}(\mathrm{K} / \mathrm{I})$, or Brilliant Cresyl Blue $(\mathrm{BCr})$. The measurements were made in water with an accuracy of $0.5 \mu \mathrm{m}$ for ascospores, asci, conidia, conidiogenous cells, conidiophores, and ascomatal and pycnidial wall cells, and $5 \mu \mathrm{m}$ for ascomata and pycnidia. The length, breadth and length/breadth ratio $(1 / b)$ of ascospores and conidia are given (when $\mathrm{n}>10$ ) as: (min-) $\{\mathrm{X}-\mathrm{SD}\}-\{\mathrm{X}+\mathrm{SD}\}(-\max )$, where "min" and " $\max$ " are the extreme observed values, $\mathrm{X}$ the arithmetic mean and SD the corresponding standard deviation. The photographs were taken with a Levenhuk C510 NG camera. All examined specimens are deposited in the lichenological herbarium of Kherson State University (KHER), in the herbarium of the Institute of Botany of the Czech Academy of Sciences (PRA) and in the private herbarium of the first author (herb. VD).

\section{RESULT}

Species newly reported for Ukraine are denoted by an asterisk (*). 


\section{*Acremonium RHABdosporum W. Gams}

The species was isolated from Cladonia thallus collected in Austria (Gams, 1971). Later, A. rhabdosporum has been observed on various corticolous lichens from Austria, the Czech Republic, Germany, the Great Britain, Luxembourg, Italy, Spain and Sweden (Gams, 1971; Hawksworth, 1979; Diederich, 1989; Santesson et al., 2004; Etayo \& López de Silanes, 2008; Brackel, 2008; 2010; Kocourková, 2009).

Specimen examined. Ukraine. Zakarpattia region, Tyachivs'kyi district, Carpathian Biosphere Reserve, Shyrokyi Luh Massive, 48¹8'26.2”N, 2343'56.9”E, alt. $830 \mathrm{~m}$, on Pseudevernia furfuracea (thallus), on Fagus sylvatica twig, 24 May 2019, V. Darmostuk (herb. VD 329).

\section{*ARthonia digitatae Hafellner}

Our specimen is characterized by very inconspicuous, convex ascomata, slightly constricted at the base, brownish epithecium, colorless hymenium, indistinct $\mathrm{K}$ reaction of hymenium, 1-septate hyaline ascospores measuring $(9.2-) 10.6-11.8(-14.6) \times(3.8-) 4.4-4.8(-6.2) \mu \mathrm{m}$ $(\mathrm{n}=30)$. These morphological features fit the concept of $A$. digitatae (Kocourková \& van den Boom, 2005; Brackel, 2015; Zhurbenko \& PinoBodas, 2017). This is a widespread holarctic species occurring also in polar desert biome (Zhurbenko \& Pino-Bodas, 2017).

Specimen examined. Ukraine. Ivano-Frankivsk region, Nadvornyans'kyi district, Nature Reserve

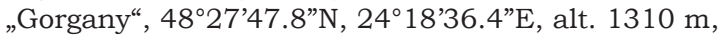
on Cladonia digitata (squamules), on wood, 27 August 2019, V. Darmostuk (KHER 13531).

\section{*Bryocentria metzGeriae (Ade \& Höhn.) Döbbeler}

This is a common in Europe bryophilous fungus growing on Radula complanata (Döbbeler, 2004, 2010).

Specimen examined. Ukraine. Zakarpattia region, Tyachivs'kyi district, Carpathian Biosphere Reserve, Shyrokyi Luh Massive, near Lushanka river, $48^{\circ} 18^{\prime} 26.2^{\prime \prime} \mathrm{N}, 23^{\circ} 43^{\prime} 56.9^{\prime \prime} \mathrm{E}$, alt. $830 \mathrm{~m}$, on Radula complanata, on Fagus sylvatica bark, 22 May 2019, A. Khodosovtsev (KHER).

\section{Clypeococcum hypocenomycis D. Hawksw.}

This is a common but yet overlooked species in Ukraine, previously recorded from Dnipropetrovsk, Lviv, Kharkiv, Kherson, Ternopil, Volyn and Zhytomyr regions (Darmostuk \& Khodosovtsev, 2017, 2020; Darmostuk \& Sira, 2020). Here it is newly reported for Ivano-Frankivsk region.
Specimen examined. Ukraine. Ivano-Frankivsk region, Nadvornyans'kyi district, Nature Reserve "Gorgany“, 48²7'47.8”N, 24¹8'36.4”E, alt. 1310 m, on Hypocenomyce scalaris (thallus), on Pinus cembra bark, 26 August 2019, V. Darmostuk (herb. VD 546).

DidymocyRTIS MELANELIXIAE (Brackel) Diederich, R.C. Harris \& Etayo

The species is probably rare in Ukraine. Recently, it has been reported on Platismatia glauca from Petros Mt. (Darmostuk, 2018).

Specimen examined. Ukraine. Zakarpattia region, Tyachivs'kyi district, Carpathian Biosphere Reserve,

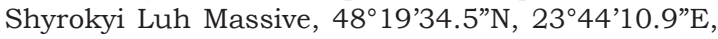
alt. $840 \mathrm{~m}$, on Melanelixia glabratula, on Fagus sylvatica bark, 22 May 2019, V. Darmostuk (herb. VD 590).

*Diplolaeviopsis cf. symmictae Diederich \& Coppins (Fig. 1)

Ascomata not observed. Conidiomata pycnidia, arising singly ( $2-5$ per host apothecium), unilocular, immersed to semi-immersed in apothecia of Lecanora symmicta, subglobose, macroscopically dark brown, 70-90 $\mu \mathrm{m}$ in diam. Pycnidial wall up to $15 \mu \mathrm{m}$ thick, composed of 2-6 layers of pseudoparenchymatous cells, aeruginous, $\mathrm{K}+$ olive brown. Conidiogenous cells subcylindrical, with a slight collarette, hyaline, smooth, (4.8-)5.2-5.6(-6.4) × (2.0-)2.2-3.0(-3.4) $\mu \mathrm{m}(\mathrm{n}=15)$. Conidia holoblastic, arising singly, elongate soleiform to tadpole-shaped, 1-septate, constricted at the septum, hyaline, smooth, $(7.2-) 7.8-9.2(-9.8) \times(2.2-) 2.8-3.6(-3.8) \mu \mathrm{m}$ $(\mathrm{n}=30)$, upper cell \pm globose, lower cell subcylindrical, sometimes equal in length with upper cell, base truncate.

There are some discrepancies between the examined specimen and the protologue (Diederich $\&$ Coppins, 2014), where conidiomata were reported as being larger, and the pycnidial wall is hyaline to olivaceous, $\mathrm{K}-$. Another species of the genus, Diplolaeviopsis ranula, is characterized by similar morphological features, but differs in its brown, $\mathrm{K}+$ purplish excipular pigment and different host species, Lecanora strobilina and $L$. strobilinoides (Giralt \& Hawksworth, 1991; Suija et al., 2015). To date, Diplolaeviopsis symmictae was reported from Europe and North America (Diederich \& Coppins, 2014; Haldeman, 2019). Specimen examined. Ukraine. Ivano-Frankivsk region, Nadvornyans'kyi district, Nature Reserve "Gorgany", $48^{\circ} 27^{\prime} 47.8^{\prime \prime} \mathrm{N}, 24^{\circ} 18^{\prime} 36.4^{\prime \prime} \mathrm{E}$, alt. $1310 \mathrm{~m}$, on Lecanora symmicta (apothecia), on Pinus cembra twig, 27 August 2019, V. Darmostuk (KHER 13446). 


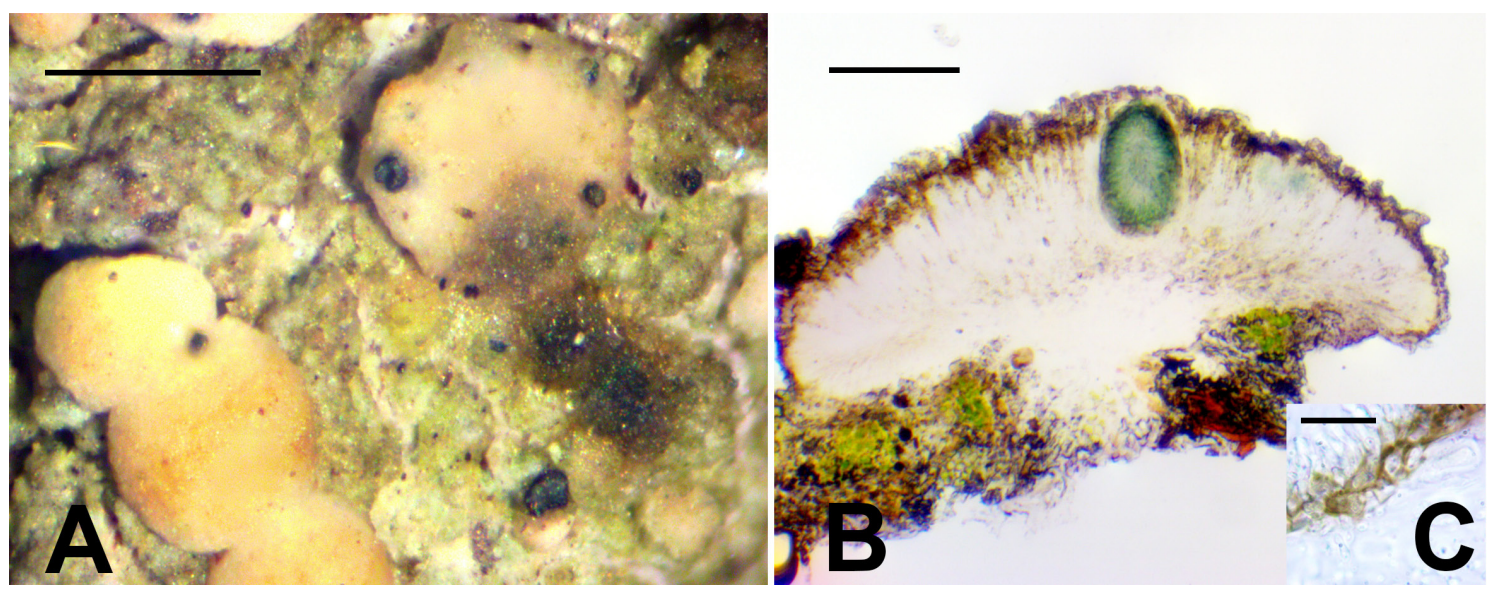

Fig. 1. Diplolaeviopsis cf. symmictae (KHER 13446): A - conidiomata on host apothecia; B - crosssection of the conidiomata; C - pycnidial wall in $\mathrm{KOH}$. Scale bars: A - 1 mm, B - $100 \mu \mathrm{m}, \mathrm{C}-10 \mu \mathrm{m}$.

\section{Epicladonia sandstedei (Zopf) D. Hawksw.}

Conidiomata semi-immersed, up to $150 \mu \mathrm{m}$ in diam., forming distinct galls, pycnidial wall orange-brown, conidia hyaline, smooth 0-1-septate (9.0-) 10.0-12.8(-14.2) × (2.2-)2.6-3.2(-3.4) $\mu \mathrm{m}(\mathrm{n}=30)$. Epicladonia sandstedei is a widely distributed species in the Holarctic (Zhurbenko \& Pino-Bodas, 2017), and it was reported in Ukraine only from Kherson region on the thallus of Cladonia rangiformis (Darmostuk \& Khodosovtsev, 2017).

Specimen examined. Zakarpattia region, Tyachivs'kyi district, Carpathian Biosphere Reserve, Shyrokyi Luh Massive, $48^{\circ} 18^{\prime} 26.2^{\prime \prime} \mathrm{N}, 23^{\circ} 43^{\prime} 56.9^{\prime \prime} \mathrm{E}$, alt. $830 \mathrm{~m}$, on Cladonia sp. on Fagus sylvatica bark at the base of trunk, 24 May 2019, A. Khodosovtsev (KHER 13013).

Epicladonia stenospora (Harm.) D. Hawksw. s.lat.

Our specimen is characterized by semi-immersed to erumpent conidiomata up to $100 \mu \mathrm{m}$ in diam., light brown pycnidial wall, hyaline smooth 0-(1)-septate conidia (8.8-)9.8-11.0($11.4) \times(2.8-) 3.0-3.4(-3.8) \mu \mathrm{m}(\mathrm{n}=30)$. Distinct gall formation was not observed. In Ukraine, it was reported from Sumy region on thalli of Cladonia foliacea (Khodosovtsev \& Darmostuk, 2017).

Specimen examined. Ukraine. Zakarpattia region, Tyachivs'kyi district, Carpathian Biosphere Reserve, Shyrokyi Luh Massive, 48²1'20.3”N, 2344'10.4"E, alt. $830 \mathrm{~m}$, on Cladonia digitata (thallus), on Picea abies bark, 26 May 2019, V. Darmostuk (herb. VD 638).
*Rhymbocarpus pubescens (Etayo \& Diederich) Diederich \& Etayo

Our material is characterized by rather immersed than erumpent ascomata with blackish hair, up to $200 \mu \mathrm{m}$ in diam., greenish grey exciple, 8-spored asci and ellipsoid 0-septate hyaline ascospore, (8.2-)8.8-9.4(-9.8) × (2.4)3.2-3.6(-4.0) $\mu \mathrm{m}(\mathrm{n}=30)$. To date, this species was reported only from Great Britain, Norway, Russia (Caucasus), Spain and Papua New Guinea (Etayo \& Diederich, 1998; Diederich \& Etayo, 2000; Santesson et al., 2004; Urbanavichus et al., 2020).

Specimen examined. Ukraine. Zakarpattia region, Tyachivs'kyi district, Carpathian Biosphere Reserve, Shyrokyi Luh Massive, Manchul, 48¹8'41.2”N, $23^{\circ} 41^{\prime} 59.7^{\prime \prime} \mathrm{E}$, alt. $1100 \mathrm{~m}$, on thallus of Lepraria incana, at the base of Fagus sylvatica, 27 May 2019, A. Khodosovtsev (KHER 13856).

*Skyttea GRegaria Sherwood, D. Hawksw. \& Coppins

This widespread species was previously reported from Austria, Finland, Germany, Great Britain, Norway, Poland, Russia, Slovenia, Spain, Sweden and USA (e.g. Diederich \& Etayo, 2000; Kukwa \& Czarnota, 2006; Stepanchikova et al., 2018).

Specimens examined (all on Violella fucata). Ukraine. Ivano-Frankivsk region, Nadvornyans'kyi district, Nature Reserve "Gorgany“, 48 $25^{\prime} 49.2^{\prime} \mathrm{N}$, $24^{\circ} 19^{\prime} 25.9$ "E, alt. $1070 \mathrm{~m}$, on Fagus sylvatica twig, 24 August 2019, V. Darmostuk (KHER 13684); Ibidem, on twig of Picea abies, J. Vondrák (PRA Vondrák 
22116); Yaremchans'ka city council, Tatariv, Polianytsa, c. $7.5 \mathrm{Km} \mathrm{NW}$ of Polianytsa, above valley of river Zubrinka, 48 24'35.7"N, 2423'41.6”'E, alt. $1300 \mathrm{~m}$, 26.08.2019, J. Vondrák (PRA Vondrák 22021); Zakarpattia region, Tyachivs'kyi district, Carpathian Biosphere Reserve, Shyrokyi Luh Massive, near river Luzhanka, 48 19'34.5”N, 2344'10.1”E, alt. 840 m, on F. sylvatica bark, 22 May 2019, A. Khodosovtsev (KHER 14118); 48¹8'26.2”N, 2343'56.9”E, alt. 830 m, on F. sylvatica bark, 24 May 2019, V. Darmostuk (KHER 13149).

\section{SPHINCTRINA ANGLiCA Nyl.}

Previously, this species was known in Ukraine from a single report [as Sphinctrina microcephala (Sm.) Nyl.] from Chernivtsi region, growing on an unidentified corticolous crustose brown lichen (Makarevich, 1955).

Specimens examined. Ukraine. Ivano-Frankivsk region, Nadvornyans 'kyi district, Nature Reserve „Gorgany“, 48 $24^{\prime} 34.7^{\prime \prime} \mathrm{N}, 2^{\circ} 23^{\prime} 42.2^{\prime} \mathrm{E}$, alt. $1310 \mathrm{~m}$, on Protoparmelia cf. hypotremella (thallus), on Betula obscura bark, 26 August 2019, J. Vondrák \& A. Khodosovtsev (KHER 13075, 13563, PRA Vondrák 22033, 22036).

\section{*Stromatopogon cladoniae Diederich \& Sérus.}

Conidiomata pycnidia, immersed, arising singly, subglobose, dark brown, 80-100 $\mu \mathrm{m}$ in diam.; pycnidial wall brown, 12-15 $\mu \mathrm{m}$ thick, conidiogenous cell hyaline, ampulliform to short cylindrical; macroconidia variable in shape, spherical, ellipsoid or obpyriform, (2-)4(-7)-septate, hyaline, thick-walled, (7.8-)9.4-13.8(-16.4) $\times$ (6.0-)6.8-8.2(-9.2) $\mu \mathrm{m}(\mathrm{n}=30)$; microconidia not observed; infected parts of squamules become decolored.

This species was reported only from Belgium (Diederich \& Sérusiaux, 2003).

Specimen examined. Ukraine. Ivano-Frankivsk region, Yaremchans'ka city council, near Zarosliak village, 48 09'48.4”N, 24³3'16.2”E, alt. $903 \mathrm{~m}$, on Cladonia sp. (thallus), on Picea abies bark, 7 August 2019, V. Darmostuk \& O. Sira (herb. VD 061).

\section{*TRemella cetrariicola Diederich \& Coppins}

This common species has been reported from Europe (Great Britain, Germany, Finland, France, Italy, Norway, Svalbard and Sweden), Asia (Russia), Canary Islands and North America (USA and Canada) (Diederich, 1996; Pippola \& Kotiranta, 2008; Zhurbenko et al., 2012; Zhurbenko \& Brackel, 2013; Brackel, 2014; Moreau et al., 2015; Brackel \& Puntillo, 2016).

Specimens examined (all on thalli Tuckermannopsis chlorophylla). Ukraine. Ivano-Frankivsk region,
Yaremchans'ka city council, near Zarosliak village, 4809'48.4"N, 24³3'16.2"E, alt. 903 m, on Picea abies bark, 7 August 2019, V. Darmostuk \& O. Sira (herb. VD 058); Zakarpattia region, Tyachivs'kyi district, Carpathian Biosphere Reserve, Shyrokyi Luh Massive, Manchul, 48 $18^{\prime} 41.2 " \mathrm{~N}, 2^{\circ} 41^{\prime} 59.7^{\prime} \mathrm{E}$, alt. $1100 \mathrm{~m}$, on Fagus sylvatica bark, 27 May 2019, V. Darmostuk (KHER 13311).

\section{XenoneCtriella fissuriprodiens (Etayo) Etayo}

It was previously reported (as Pronectria fissuriprodiens Etayo) from Zakarpattia region without certain locality (Kondratyuk et al., 2003).

Specimen examined. Ukraine. Zakarpattia region, Tyachivs'kyi district, Carpathian Biosphere Reserve, Shyrokyi Luh Massive, 48¹9'34.5”N, 2344'10.1"E, alt. 840 m, on Lobaria pulmonaria, on Fagus sylvatica, 22 May 2019, A. Khodosovtsev (KHER 14172).

\section{*Xenonectriella subimperspicua (Speg.) Etayo}

Ascomata perithecia, immersed in host thalli, globose, orange, arising singly or in groups of 2-4, 100-150 $\mu \mathrm{m}$ in diam.; exciple up to 20 $\mu \mathrm{m}$ thick, $\mathrm{K}+$ violet; asci cylindrical, 8-spored (36-)42-49(-52) × (5.5-)8-9.2(-10) $\mu \mathrm{m}(\mathrm{n}=10)$; ascospores 1 -septate, broadly ellipsoid, hyaline, constricted at the septum, (6.2-)6.8-7.8(-8.0) $\times(4.4-) 4.8-5.4(-5.6) \mu \mathrm{m}(\mathrm{n}=30)$. This species was previously reported on various species of Parmeliaceae and Physciaceae from Argentina, Brazil, Ecuador, Germany, India, New Zealand and Spain (Brackel, 2007; Zhurbenko, 2013; Motiejūnaitė \& Grochowski, 2014; Etayo \& PérezOrtega, 2016; Etayo, 2017). Parmelia saxatilis and Parmelina pastillifera are new host species.

Specimen examined. Ukraine. Zakarpattia region, Tyachivs'kyi district, Carpathian Biosphere Reserve, Shyrokyi Luh Massive, Manchul, 48 18'41.2"N, 23 41'59.7"E, alt. $1100 \mathrm{~m}$, on Parmelina pastillifera and Parmelia saxatilis (thallus), on Fagus sylvatica, 27 May 2019, A. Khodosovtsev (KHER 13385).

\section{ACKNOWLEDGMENTS}

We express our gratitude to Wolfgang von Brackel, Michael Haldeman and Jurga Motiejūnaitè for kindly providing the literature and Nataliya Maluga for help with herbarium management (KHER). Jiři Malíček, Zdeněk Palice, Ondřej Peksa, Yaroslav Petraschuk, Stanislav Svoboda and Olexandr Yurchenko were of indispensable assistance during fieldwork excursions. The anonymous reviewer and Tiina Randlane (Tartu) are thanked for their valuable comments and 
suggestions. This study was financially supported by the Ministry of Science and Education of Ukraine (Project No 0219U000270).

\section{REFERENCES}

Brackel, W. v. 2007. Weitere Funde von flechtenbewohnenden Pilzen in Bayern Beitrag zu einer Checkliste III. Berichte Der Bayerischen Botanischen Gesellschaft 77: 5-26.

Brackel, W. v. 2008. Zwackhiomyces echinulatus sp. nov. and other lichenicolous fungi from Sicily, Italy. Herzogia 21: 181-198.

Brackel, W. v. 2010. Weitere Funde von flechtenbewohnenden Pilzen in Bayern - Beitrag zu einer Checkliste V. Berichte Der Bayerischen Botanischen Gesellschaft 80: 5-32.

Brackel, W. v. 2014. Kommentierter Katalog der flechtenbewohnenden Pilze Bayerns. Bibliotheca Lichenologica 109: 1-476.

Brackel, W. v. 2015. Lichenicolous fungi from Central Italy with notes on some remarkable hepaticolous, algicolous and lichenized fungi. Herzogia 28(1): 212-281. https://doi.org/10.13158/ heia.28.1.2015.212

Brackel, W. v. \& Puntillo, D. 2016. New records of lichenicolous fungi from Calabria (Southern Italy), including a first checklist. Herzogia 29(2): 277-306. https://doi.org/10.13158/ heia.29.2.2016.277

Coppins, B. J., Kondratyuk, S. Ya., Khodosovtsev, A. Ye., Zelenko, S. D., Coppins A. M., Wolseley P. A. \& Virchenko V. M. 1998. Diversity of Lichens and Bryophytes in Regional Landscape Park „Stuzhytzia” (Ukrainian part of the International Biosphere Reserve „Eastern Carpathians”). In: Lobarion lichens as indicators of the primeval forests of the Eastern Carpathians (Darwin International Workshop, 25-30 May 1998, Kostrino, Ukraine), Kostrino. Pp. 139-161.

Darmostuk, V. V. 2018. The new records of lichenicolous fungi from Ukrainian Carpathians. Chornomorski Botanical Journal 14(2): 173-179. (In Ukrainian). https: / / doi.org/ 10.14255/23089628/18.142/7

Darmostuk, V. V. \& Khodosovtsev, A. Ye. 2017. Lichenicolous fungi of Ukraine: An annotated checklist. Studies in Fungi 2(1): 138-156. https:// doi.org/10.5943/sif/2/1/16

Darmostuk, V. V. \& Khodosovtsev, A. Ye. 2020. Notes to lichen-forming and lichenicolous fungi in Ukraine I. Chornomorski Botanical Journal 16(3): 257-274. https://doi.org/10.32999/ ksu1990553X/2020-16-3-6

Darmostuk V. V. \& Sira O. Ye. 2020. New and remarkable records of lichenicolous fungi from Ternopil Oblast (Ukraine). Czech Mycology 72(1): 33-41. https://doi.org/10.33585/cmy.72103

Diederich, P. 1989. Les lichens epiphytiques et leurs champignons lichenicoles (macrolichens exceptes) du Luxembourg. Travaux Scientifiques du Musee National D'Histoire Naturelle de Luxembourg 14: 1-268.

Diederich, P. 1996. The lichenicolous heterobasidiomycetes. Bibliotheca Lichenologica 61: 1-198.

Diederich, P. \& Coppins, B. 2014. Diplolaeviopsis symmictae (Helotiales, Ascomycota), a new lichenicolous fungus on Lecanora symmicta. Bulletin de La Société Des Naturalistes Luxembourgeois 115: 151-155.

Diederich, P. \& Etayo, J. 2000. A synopsis of the genera Skyttea, Llimoniella and Rhymbocarpus (Lichenicolous Ascomycota, Leotiales). The Lichenologist 32(05): 423-485. https://doi. org/10.1006/lich.2000.0290

Diederich, P. \& Sérusiaux, E. 2003. Stromatopogon cladoniae sp. nova, a remarkable new lichenicolous coelomycete from Belgium. Bibliotheca Lichenologica 86: 103-106.

Döbbeler, P. 2004. Bryocentria (Hypocreales), a new genus of bryophilous Ascomycetes. Mycological Progress 3(3): 247-256. https:/ / doi.org/10.1007/ s11557-006-0095-7

Döbbeler, P. 2010. New species and records of Bryocentria - a hypocrealean genus of bryophilous ascomycetes. Karstenia 50: 11-23. https://doi. org/10.29203/ka.2010.437

Etayo, J. 2017. Hongos liquenícolas de Ecuador. San Miguel de Tucumán. 540 pp.

Etayo, J. \& Diederich, P. 1998. Lichenicolous fungi from the Western Pyrenees, France and Spain. IV. Ascomycetes. The Lichenologist 30: 103-120. https://doi.org/10.1006/lich.1997.0121

Etayo, J. \& López de Silanes, M. E. 2008. Liquenes epífitos y hongos liquenicolas del Bosque Viejo de Munain-Okariz (Álava, País Vasco, España). Nova Acta Científica Compostelana (Bioloxía) 17: 11-29.

Etayo, J. \& Pérez-Ortega, S. 2016. Lichenicolous lichens and fungi from Monfragüe National Park (Western Spain). Herzogia 29(2): 315-328. https://doi.org/10.13158/heia.29.2.2016.315

Gams, W. 1971. Cephalosporium-artige Schimmelpilze (Hyphomycetes). Stuttgart. 262 pp.

Giralt, M. \& Hawksworth, D. L. 1991. Diplolaeviopsis ranula, a new genus and species of lichenicolous coelomycetes growing on the Lecanora strobilina group in Spain. Mycological Research 95(6): 759-761. https://doi.org/10.1016/S09537562(09)80828-9

Haldeman, M. 2019. New and interesting records of lichens and lichenicolous fungi from Northwestern USA II. Evansia 36(3): 63-73. https://doi. org/10.1639/0747-9859-36.3.63

Hawksworth, D. L. 1979. The lichenicolous Hyphomycetes. Bulletin of the British Museum of Natural History 6: 183-300.

Hawksworth, D. L. 1981. The lichenicolous Coelomycetes. Bulletin of the British Museum for Natural History 9(1): 1-98.

Khodosovtsev, A. Ye. \& Darmostuk, V. V. 2017. Zwackhiomyces polischukii sp. nov., and other 
noteworthy lichenicolous fungi from Ukraine. Polish Botanical Journal 62(1): 27-35. https:// doi.org/10.1515/pbj-2017-0006

Kocourková, J. 2009. Observations on the genus $\mathrm{Ne}$ olamya, with the description of the new species N. xanthoparmeliae (Ascomycota, genera incertae sedis). Opuscula Philolichenum 6: 137-148.

Kocourková, J. \& van den Boom, P. 2005. Lichenicolous fungi from the Czech Republic II. Arthrorhaphis arctoparmeliae spec. nov. and some new records for the country. Herzogia 18: 23-35.

Kondratyuk, S. Ya., Popova, L. P., Lackovicova, A. \& Pišút, I. 2003. A catalogue of the Eastern Carpathian Lichens. Kiev-Bratislava. 263 pp.

Kukwa, M. \& Czarnota, P. 2006. New or interesting records of lichenicolous fungi from Poland IV. Herzogia 19: 111-123. https://doi.org/10.13158/ heia.23.1.2010.111

Makarevich, M. F. 1955. To study of lichen flora of Chernivtzi oblast. Botanical Zhurnal of Academy of Sciences of USSR 12(2): 52-59.

Malíček, J., Palice, Z., Acton, A., Berger, F., Bouda, F., Sanderson, N. \& Vondrák, J. 2018. Uholka primeval forest in the Ukrainian Carpathians a keynote area for diversity of forest lichens in Europe. Herzogia 31(1): 140-171. https://doi. org/10.13158/099.031.0110

Moreau, P.-A., Haluwyn, C. V., Roux, C. \& Sussey, J.-M. 2015. Tremella cetrariicola Diederich et Coppins, première récolte en France. Bulletin d'informations de l'Association Française de Lichénologie 40(2): 163-167.

Motiejūnaitè, J. \& Grochowski, P. 2014. Miscellaneous new records of lichens and lichenicolous fungi. Herzogia 27(1): 193-198. https://doi. org/10.13158/heia.27.1.2014.193

Nyporko, S. O., Barsukov, O. O. \& Kapets, N. V. 2018. Floristic records of mosses, lichens and lichenicolous fungi from Hutsulschyna National Nature Park. Ukrainian Botanical Journal 75(2): 179-186. https://doi.org/ 10.15407/ukrbotj75.02.179

Pippola, E. \& Kotiranta, H. 2008. The genus Tremella (Basidiomycota, Tremellales) in Finland. Annales Botanici Fennici 45: 401-434. https://doi. org/10.5735/085.045.0601

Santesson, R., Moberg, R., Nordin, A., Tønsberg, T. \& Vitikainen, O. 2004. Lichen-forming and licheni- colous fungi of Fennoscandia. Uppsala. 359 pp.

Servit, M. 1936. Neue und seltenere Flechten aus den Familien Verrucariaceae und Dermatocarpaceae. Beihefte zum botanischen Centralblatt 55: 251-274.

Servit, M. \& Nádvorník, J. 1932. Flechten aus der Čechoslovakei. II. Karpatorussland und Südostslovakei. Věstník Králouské české společnosti náuk. Trüda mathematicko-prĭrodovédecká. Pp. $1-41$.

Stepanchikova, I. S., Himelbrant, D. E., Motiejūnaitè, J., Ahti, T., Suija, A., Kuznetsova, E. S. \& Dyomina, A. V. 2018. New records of lichens and allied fungi from the Leningrad Region, Russia. IX. Folia Cryptogamica Estonica 55: 117-124. https:/ / doi. org/ 10.12697 / fce.2018.55.12

Suija, A., Ertz, D., Lawrey, J. D. \& Diederich, P. 2015. Multiple origin of the lichenicolous life habit in Helotiales, based on nuclear ribosomal sequences. Fungal Diversity 70(1): 55-72. https://doi. org/10.1007/s13225-014-0287-4

Urbanavichus, G., Vondrák, J., Urbanavichene, I., Palice, Z. \& Malíček, J. 2020. Lichens and allied non-lichenized fungi of virgin forests in the Caucasus State Nature Biosphere Reserve (Western Caucasus, Russia). Herzogia 33: 90-138. https:/ / doi.org/10.13158/heia.33.1.2020.90

Zhurbenko M. P. 2013. A first list of lichenicolous fungi from India. Mycobiota 3: 19-34. https:// doi.org/10.12664/mycobiota.2013.03.03

Zhurbenko, M. P. \& Brackel, W. v. 2013. Checklist of lichenicolous fungi and lichenicolous lichens of Svalbard, including new species, new records and revisions. Herzogia 26(2): 323-359. https://doi. org/10.13158/heia.26.2.2013.323

Zhurbenko, M. P., Himelbrant, D. E., Kuznetsova, E. S. \& Stepanchikova, I. S. 2012. Lichenicolous fungi from the Kamchatka Peninsula, Russia. The Bryologist 115(2): 295-312. https://doi. org/10.1639/0007-2745-115.2.295

Zhurbenko, M. P. \& Pino-Bodas, R. 2017. A revision of lichenicolous fungi growing on Cladonia, mainly from the Northern Hemisphere, with a worldwide key to the known species. Opuscula Philolichenum 16: 188-266. 